\title{
Dangers of glycerol therapy for acute glaucoma after a partial gastrectomy
}

\author{
A. W. SOLLOM \\ Wolverhampton, Staffordshire
}

The introduction of the osmotic agents has dramatically altered the management of patients suffering from acute closed-angle glaucoma. Intravenous therapy is available as urea, mannitol, and sodium ascorbate, but the only oral preparation in general use is glycerol. The commonly recognized complications are mild headache, pain in the neck, and nausea and vomiting, and there is the added risk of thrombophlebitis with the intravenous agents. The purpose of this report is to draw attention to another obvious but apparently unrecorded problem.

\section{Case report}

A woman aged 49 presented with a typical history of attacks of subacute angle-closure glaucoma which had continued for several months. The angles were closed and the raised pressure did not completely respond to miotics and diamox, so that an early drainage operation was planned.

In preparation for this she was given oral glycerol, at the dosage rate of $1.5 \mathrm{~g} . / \mathrm{kg}$. body weight, in fruit juice. It was known that she had undergone a partial gastrectomy for duodenal ulcer 3 years previously.

There was a most severe reaction to this drink and within half an hour she had developed a very severe headache and prostration such that at one stage it was considered advisable to postpone surgery. However, she improved spontaneously and the operation was performed. Shortly after its completion, however, she experienced a severe attack of diarrhoea, which was not repeated. By the next day all the symptoms had subsided. There were no other unexpected events and she made good progress.

\section{Discussion}

One of the well-recognized complications of partial gastrectomy is the dumping syndrome. This term is used when the rapid passage of food into the small intestine results in the presence of an irritant hypertonic solution in the jejunum. It is thought that the abrupt distention of the postanastomotic small intestine causes transudation from the jejunal wall and a sudden fall of plasma volume. This leads to symptoms of sweating and faintness, with nausea or sometimes collapse. The faeces may be soft or watery indicating hypermotility. It seems very likely that this patient suffered from a similar phenomenon. The rapid passage of the glycerol into the small intestine led to its rapid absorption followed by the severe effects described above.

Glycerol was introduced into ophthalmology as a means of controlling glaucoma by Virno, Gantore, Bietti, and Bucci ( 1963 ), and it is recommended for use in a dose of $0 \cdot 75^{-1} \cdot 5 \mathrm{~g} . / \mathrm{kg}$. prepared as a $5^{0}$ per cent. solution. It reaches a maximum effect after one hour but it continues to act for 4 to 6 hours. Casey and Trevor-Roper (1963) found that the fall of intraocular pressure was directly related to the rise of serum osmolarity. 
Buckell and Walsh (1964) studied the effect of glycerol on raised intracranial pressure. They found that, if the glycerol was sipped with an equal volume of iced lemon juice over a 20-minute period, then the plasma osmolarity rose 25 per cent. more than after the equivalent dose of oral urea, but that the diuresis was less. They also found that there were no toxic effects after administering glycerol for many weeks two or three times daily. Some nausea and even vomiting are experienced by many patients, probably because of its irritant effect on the stomach. These symptoms can be minimized by the prior administration of an antiemetic.

Glycerol is a bivalent alcohol which has a calorific value slightly greater than glucose. It is metabolized in a similar way and it follows that it must be used with care in diabetic patients. The onset of diabetic coma after the administration of oral glycerol has been recorded (D'Alena and Ferguson, I 966). All the osmotic agents may cause some headache and pain in the back, and even mental confusion occurs in some patients (Thomas, 1963; Drance, 1964). This is probably caused by the temporary reduction in the extracellular fluid volume as this is drawn into the circulation by the process of osmosis. A slight shrinkage of cerebral tissue then occurs and causes traction on pain sensitive arteries or venous sinuses. The increased blood volume which results from the osmotic effect may embarass a failing heart while the infusion of the intravenous agents (usually $500 \mathrm{ml}$.) may be sufficient to provoke congestive cardiac failure. The increased circulating volume may lead to severe sodium depletion in advanced renal disease when there is a limited reabsorption of sodium salts under normal conditions. The use of urea is particularly contraindicated in renal failure as it increases nitrogen retention. Glycerol takes part in the formation of body fat, as well as in carbohydrate metabolism. It is therefore theoretically possible that, in cases of advanced liver disease, these metabolic pathways may be defective and so lead to the prolongation of the osmotic effect. Some of the side-effects are potentially dangerous, and in the presence of contraindications the drug should be avoided or used in a much reduced dosage. The postgastrectomy patient should be added to the list of conditions in which caution is required, and it is probable that care is also needed in dealing with other intestinal malabsorption syndromes.

\section{Summary}

Severe headache, prostration, and diarrhoea followed the administration of oral glycerol to a patient who had undergone partial gastrectomy. The mechanism of the reaction is discussed and a reduced dosage is recommended in this group of patients.

\section{References}

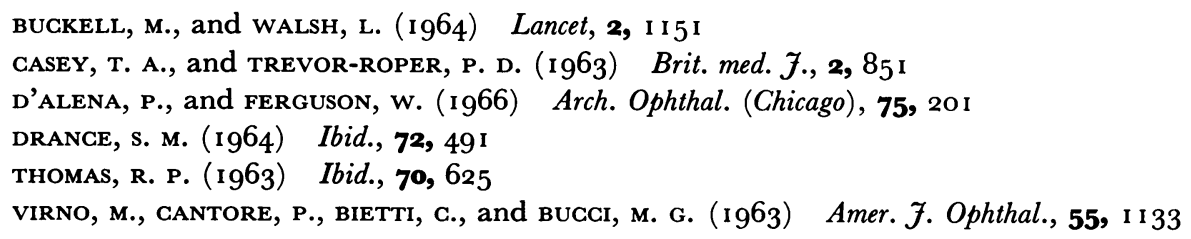

